\title{
INGRESO DE ESTUDIANTES TRANS A ESTABLECIMIENTOS EDUCACIONALES CHILENOS: ANÁLISIS DE TOPOI EN COMENTARIOS DE FACEBOOK $^{1}$
}

\section{ADMISSION OF TRANS STUDENTS TO CHILEAN EDUCATIONAL INSTITUTIONS: TOPOI ANALYSIS ON FACEBOOK COMMENTS}

\author{
Paulina Maldonado Delgado \\ Universidad de Concepción, Chile \\ paumaldonado.d@gmail.com \\ Paola Alarcón Hernández \\ Universidad de Concepción, Chile \\ palarco@udec.cl \\ Carolina Venegas Carrasco \\ Universidad de Concepción, Chile \\ carovenegasc@udec.cl \\ Mabel Urrutia Martínez \\ Universidad de Concepción, Chile \\ maurrutia@udec.cl
}

\section{Resumen:}

El objetivo de la investigación es analizar los topoi en los que se basan las posiciones a favor y en contra del ingreso de estudiantes trans a establecimientos educacionales chilenos. El estudio se inscribe, de manera general, en la Lingǘstica Cognitiva y recoge, desde allí, la propuesta de Wodak sobre la estrategia discursiva de los topoi como forma de justificar determinadas posturas hacia las personas trans. El corpus está conformado por comentarios digitales posteados por lectores/as en las páginas de Facebook de los medios chilenos Chilevisión, CNN Chile, Cooperativa, en 2019. El procedimiento siguió las etapas: a) preanálisis, b) codificación, c) categorización. Los resultados muestran un predominio de comentarios que manifiestan una actitud negativa hacia personas trans y LGBTIQ + , a través de seis topoi, los que contribuyen a la construcción y circulación de representaciones sociales en nuestra sociedad.

Palabras clave: personas trans, sistema escolar, topoi, representación social, comentario digital.

\footnotetext{
${ }^{1}$ Esta investigación fue financiada por el Fondo Nacional de Desarrollo Científico y Tecnológico (Chile) a través del proyecto FONDECYT Regular N 1201451 "Un estudio de las representaciones discursivas de las personas LGBTIQ+ desde la lingüística cognitiva".
} 
Artículo. Paulina Maldonado Delgado, Paola Alarcón Hernández, Carolina Venegas Carrasco, Mabel Urrutia Martínez. "Ingreso de estudiantes trans a establecimientos educacionales chilenos:

análisis de topoi en comentarios de Facebook"

\begin{abstract}
:
The objective of this research is to analyze the topoi on which both stances for and against the admission of trans students to Chilean educational institutions are based. This study is inscribed, in general terms, in Cognitive Linguistics and addresses, from that point, Wodak's proposal about the discursive strategy of the topoi as a form to justify certain stances towards trans people. The corpus is composed by digital comments posted by readers on Facebook pages of following Chilean media during 2019: Chilevisión, CNN Chile and Cooperativa. The procedure followed these stages: a) pre-analysis, b) coding, c) categorization. The results show a predominance of comments that manifest a negative attitude towards trans and LGBTIQ+ people, through the six topoi, which contribute to the construction and circulation of social representations in our society.
\end{abstract}

Keywords: trans people, school system, topoi, social representations, digital comments.

Recibido: 30 de septiembre de 2021

Aceptado: 09 de noviembre de 2021

\title{
1. Introducción
}

Durante la última década, en Chile se han tomado medidas a nivel nacional para otorgar mayor visibilidad a la población trans. En el contexto educativo, el año 2017 comenzó a regir la ODR Nº768 de la Superintendencia de Educación que define los derechos de niños, niñas y adolescentes trans en el ámbito de la educación. Esta circular apunta hacia el reconocimiento y la protección de la identidad de género de los/as estudiantes en los establecimientos, basada en la dignidad del ser humano, el interés superior de niños, niñas y adolescentes, la no discriminación arbitraria y la buena convivencia escolar. Luego, durante el año 2019, ocurrieron hitos importantes en materia de derechos de estudiantes transgénero, debido a que instituciones educativas de relevancia histórica a nivel nacional, como el Liceo $\mathrm{n}^{\circ} 1$ Javiera Carrera y el Instituto Nacional, aceptaron por primera vez a estudiantes trans reconociendo y respetando tanto su identidad como expresión de género. Además, el colegio Rosa Ester Alessandri Rodríguez, tras amplias discusiones dentro de la comunidad escolar, se convirtió en el primer liceo multigénero del país.

Sin embargo, a pesar de las medidas implementadas a nivel nacional, la realidad vivida por estudiantes trans, y LGBTIQ+ en general, dentro del sistema educativo dista mucho del ideal de una educación libre de discriminación. Así lo revela el XVIII Informe 
Anual de Derechos Humanos de la Diversidad Sexual y de Género en Chile (Movilh, $X V I I I)$, con respecto a la discriminación educacional, en el que se expone que las denuncias por discriminación a estudiantes LGBTIQ+ en el año 2019 aumentaron en un 5,4\%, ascendiendo a 39 episodios abusivos, de carácter homo y transfóbico, denunciados. Luego, en el año 2020, según el XIX Informe Anual de Derechos Humanos de la Diversidad Sexual y de Género en Chile (Movilh, XIX), a pesar de la ausencia de clases presenciales impulsadas por el contexto de crisis sanitaria que se vive a nivel mundial, seis instituciones educativas fueron responsables de casos de discriminación educacional de carácter trans y homofóbico. Esto se traduce en que, incluso existiendo un contacto mínimo entre actores educativos y estudiantes, las conductas discriminatorias permanecen.

En este sentido, consideramos de suma importancia abordar la comunicación a través de medios digitales, ya que durante el siglo XXI este tipo de interacción ha cobrado gran relevancia para la vida en sociedad, situación que se vio acrecentada mundialmente desde fines de 2019. A partir de entonces, la implementación de las TIC (Tecnologías de la Información y la Comunicación) trascendió a múltiples esferas de la vida cotidiana de las personas, tanto en el ámbito personal y familiar, como laboral y/o académico.

En consecuencia, es un hecho que el uso masivo de redes sociales -Instagram, Facebook o Tiktok, como algunos ejemplos- ha cobrado un papel importante en la interacción social de este siglo y, por lo tanto, ha dado paso al surgimiento de nuevas formas de comunicación. Autores como Sal Paz conceptualizan el lugar en que se produce la interacción online, denominado ciberespacio, como "un entorno o escenario sin cuerpo y sin geografía, delimitado por las prácticas y las presencias construidas" (Comentario 154), lo que implica que la mayor relevancia de ciberespacio radica en su aspecto relacional e interaccional, es decir, en los hechos sociales que en él ocurren.

Este estudio se inscribe, de manera general, en la Lingüística Cognitiva (LC), enfoque en el que, en la actualidad, se observa un interés creciente por integrar las perspectivas cognitiva y social, en lo que se ha llamado el giro social de la LC (Romano y Porto; Dąbrowska y Divjak; Croft). En este marco, este trabajo atiende a la dimensión socio-cognitiva del discurso (Romano y Porto; Landau, Robinson y Meier; Van Dijk), en el entendido de que las representaciones se construyen social y discursivamente en el curso de la comunicación en un contexto dado y que contribuyen a establecer identidades y 
Artículo. Paulina Maldonado Delgado, Paola Alarcón Hernández, Carolina Venegas Carrasco, Mabel Urrutia Martínez. "Ingreso de estudiantes trans a establecimientos educacionales chilenos: análisis de topoi en comentarios de Facebook"

relaciones sociales en el flujo de la comunicación. Desde esta perspectiva, el estudio que presentamos aborda las representaciones sociales de las personas trans mediante el análisis de la estrategia discursiva de los topoi identificadas en comentarios de Facebook.

Es por esto que, teniendo en cuenta los acontecimientos del año 2019 y la gran relevancia que ha adquirido la interacción digital, el objetivo de esta investigación es analizar los principales topoi en los que se basan las posiciones a favor y en contra del ingreso de estudiantes trans a establecimientos educacionales, presentes en comentarios relativos a noticias publicadas por medios de prensa chilenos en sus páginas de Facebook el año 2019.

\section{Marco teórico}

Desde un enfoque histórico del discurso, Wodak (104) plantea que el lenguaje, ya sea en su forma escrita o hablada, representa una práctica social. Consecuentemente, considera el discurso como la forma de otorgar significado, desde una perspectiva específica, a un ámbito de la práctica social. Además, cataloga como dialéctica la relación que se establece entre las prácticas discursivas y los ámbitos de acción en que se enmarcan. Esto debido a que, así como los discursos se configuran bajo determinaciones sociales, situacionales e institucionales, inevitablemente son estos mismos los que influyen en acciones y procesos político-sociales, tanto de carácter discursivo como no discursivo. En palabras de Ruth Wodak, "los discursos, en tanto que prácticas sociales lingüísticas, pueden considerarse como elementos que constituyen prácticas sociales discursivas y no discursivas, y, al mismo tiempo, como elementos constituidos por ellas" (105).

Sobre la relación dialéctica en la que las situaciones, instituciones y estructuras sociales dan forma al evento discursivo y viceversa, Ramírez y Valencia comparten la visión de Wodak con respecto a que el discurso es una práctica social definiéndolo como:

un evento comunicativo específico que involucra una cantidad de actores sociales, esencialmente en los roles del hablante/escribiente y oyente/lector (pero también en otros roles, como observador o escucha), que intervienen en un acto comunicativo, en una situación específica (tiempo, lugar, circunstancias) y del contexto. (29) 
Con el objetivo de analizar discursos políticos y discriminatorios, Wodak propone, desde lo que ella describe como un punto de vista abstracto, algunas herramientas analíticodiscursivas que considera especialmente útiles. Estos instrumentos, denominados estrategias discursivas, corresponden según la autora a un "plan de prácticas más o menos preciso y más o menos intencional (incluyendo las prácticas discursivas) que se adopta con el fin de alcanzar determinado objetivo social, político, psicológico o lingüístico" (115).

\subsection{Topoi como recursos argumentativos}

Una de las estrategias discursivas descritas por Ruth Wodak hace referencia a los argumentos mediante los cuales se busca justificar y legitimar las formas positivas o negativas de referirse a una persona o grupo. En estas estrategias de argumentación, el recurso utilizado son los topoi, definidos por la autora como "elementos de la argumentación que forman parte de las premisas obligatorias, ya tengan estas un carácter explícito o precisen inferencia" (115). Wodak adopta el término desde la teoría de la argumentación de Ascombre y Ducrot, quienes describen los discursos argumentativos poniendo especial énfasis en el encadenamiento de dos segmentos, donde una afirmación, presentada como argumento, justifica una conclusión. En este sentido, Ascombre especifica que los topoi consisten en principios ideológicos compartidos por una comunidad lingüística que "si bien sirven para la construcción arbitraria de representaciones ideológicas, se presentan siempre como si fueran exteriores al locutor, y por consiguiente, totalmente objetivos" (301).

Ascombre y Ducrot afirman que algunas de las características de los topoi es que son comunes, debido a que suponen una creencia compartida por cierta colectividad o al menos por los interlocutores de un discurso incluso antes de que este mismo se emplee, y generales en la medida en que un topos podría no ser exclusivo de la situación particular en que se está utilizando, sino que puede aplicarse en diferentes contextos discursivos. Es por esto que Wodak considera que los topoi constituyen un recurso para examinar diferentes formas de discriminación provocadas, por ejemplo, por el nacionalismo, el etnicismo y el racismo, teniendo en cuenta los argumentos tanto a favor como en contra de estos tipos de exclusión social. 
Artículo. Paulina Maldonado Delgado, Paola Alarcón Hernández, Carolina Venegas Carrasco, Mabel Urrutia Martínez. "Ingreso de estudiantes trans a establecimientos educacionales chilenos: análisis de topoi en comentarios de Facebook"

En concordancia con lo anterior, Ibarra (66) concibe los topoi como significados recurrentes o lugares comunes en la argumentación. Esto implica que se trata de creencias comunes, validadas por una comunidad y consideradas verdades suficientes para aceptar una conclusión. Es decir, son conocimientos considerados compartidos por los hablantes y utilizados como justificación para defender un punto de vista determinado.

\subsection{Representaciones sociales y discursivas}

Desde la psicología social, las representaciones sociales pueden ser comprendidas como conocimientos colectivos, comunes en un grupo social, que estructuran y dan sentido a la realidad y, por tanto, a las actitudes y comportamientos de los individuos. Esta noción fue originalmente abordada por el Moscovici, quien a pesar de estimar que una definición muy precisa podría limitar el alcance conceptual de las representaciones sociales, las considera:

entidades casi tangibles. Circulan, se cruzan y se cristalizan sin cesar en nuestro universo cotidiano a través de una palabra, un gesto, un encuentro. La mayor parte de las relaciones sociales estrechas, de los objetos producidos o consumidos, de las comunicaciones intercambiables están impregnadas de ellas. Sabemos que corresponden, por una parte, a la sustancia simbólica que entra en su elaboración y, por otra, a la práctica que produce dicha sustancia, así como la ciencia o los mitos corresponden a una práctica científica y mítica. (27)

Desde la Lingüística Cognitiva, Koller (20) las entiende como representaciones socio-cognitivas, las que comprenden creencias y/o conocimientos, incluido el conocimiento de segunda mano adquirido a través del consumo de medios, las normas y valores de los miembros de una comunidad de discurso, las actitudes y expectativas derivadas de la combinación de creencias/conocimientos, por un lado, y normas y valores, por el otro, y las emociones que se acumulan en todos estos elementos. Esta concepción coincide con la de Augoustinos et al. dado que en las representaciones sociales se combinan estructuras afectivas con dimensiones normativas y evaluativas inherentes (173). Se destaca también el rol de la comunicación en la circulación y conformación de las 
representaciones. Koller postula que la cognición crea una instancia de discurso, a la vez que es moldeada por él, lo cual implica que las representaciones están culturalmente ligadas y que ocurren en determinados momentos históricos.

Coincidimos con Ramírez (45) y Montecino (333) en que las representaciones sociales se actualizan en representaciones discursivas; de esta forma, mediante el uso de diversos recursos lingüísticos, en contextos específicos de enunciación, los hablantes van construyendo y negociando formas de representar el mundo. En la misma línea, Meneses (260) sostiene que la construcción de una representación verbal en cuanto enunciación depende de sus representaciones sociales, con la consideración de que, si bien los seres humanos estamos situados e insertos en un contexto histórico-social, seguimos manteniendo la posibilidad del cambio y de transformación social. De esta forma, las representaciones sociales no son estáticas sino que se modifican y es aquí donde las manifestaciones expresivas desempeñan un rol crucial pues no solo permiten visibilizar las representaciones sociales sino que pueden contribuir a modificarlas.

\subsection{El género comentario digital}

El aumento de la participación de la ciudadanía en los medios de comunicación digitales ha favorecido el derecho a expresión mediante la habilitación de espacios de opinión en sus propios sitios y/o a través de las publicaciones de estos mismos contenidos en los servicios de redes sociales (SRS). En este contexto, esta investigación incorpora el rol que tienen los comentarios de Facebook en la conformación de representaciones discursivas de personas LGBTQ+.

El comentario digital es definido por Sal Paz como "el género interactivo prototípico de los 'nuevos medios', unidad terminológica que aglutina diferentes manifestaciones periodísticas (diarios virtuales, weblogs, redes sociales, entre otros) emanadas en el seno de la cibercultura" (Comentario 152). Uno de los rasgos característicos de los cibermedios, además de la hipertextualidad y la multimedialidad, es la interactividad, entendida como "la posibilidad que ofrecen las plataformas online a los usuarios para que comenten, modifiquen o produzcan información a través de herramientas digitales puestas a su disposición por un medio, una red o una plataforma tecnológica” (García y Gutiérrez 296). 
Artículo. Paulina Maldonado Delgado, Paola Alarcón Hernández, Carolina Venegas Carrasco, Mabel Urrutia Martínez. "Ingreso de estudiantes trans a establecimientos educacionales chilenos: análisis de topoi en comentarios de Facebook"

El comentario digital es un género dialógico, que remite a discursos previos y en el cual los roles de emisor y de receptor son intercambiables (Sal Paz, El comentario 164). La interacción puede ser simultánea o sucesiva, pero siempre como respuesta a una voz anterior: la entrada original o algún otro comentario (De Piero y Narvaja 761).

Cantamutto y Vela proponen una caracterización del discurso digital, la que aborda tres grupos: modo de realización, enunciación y relaciones interpersonales, cada uno con sus respectivos binomios. Destacamos para fines de nuestro estudio, en el modo de realización, el binomio monomodal/multimodalidad, dado que, como señalan las autoras, el discurso digital tiende hacia la multimodalidad, no obstante lo cual "puede establecerse una gradación en función de si se inclina hacia la monomodalidad o hacia un enriquecimiento multimodal" (305). En el caso de los comentarios de Facebook, se observa que estos varían según el grado de mono/multimodalidad que se identifica en las intervenciones. Se encuentran intervenciones monomodales, en las cuales se usa un único código semiótico o multimodales, en las que se combinan códigos. En cuanto al binomio visual/verbal, las interacciones se describen de acuerdo con el código que usan: serán verbales cuando el código sea lingüístico y visuales cuando utilicen otros códigos semióticos, como imágenes, sean fijas o en movimiento. Cuando hay más de uno, es posible que uno prevalezca. Asimismo, las autoras plantean que "la relación entre ambos puede darse a modo de anclaje, el código lingüístico sobre el visual (Barthes, 1970) o a modo de ilustración, el visual sobre el verbal" (306). En relación con los signos no verbales, se identifican formas de semiosis visuales, en las que se incluyen imágenes fijas como fotografías, y en movimiento (gifs, videos), “y también cuestiones paralingüísticas que pueden estar iconizadas (el "me gusta" en Facebook) o pueden estar ocurriendo (p. e., gestos en las video-llamadas)" (306).

\section{Metodología}

El estudio se inscribe en el paradigma interpretativo o cualitativo de investigación. Según Hernández, Fernández y Baptista, el paradigma interpretativo se centra en el estudio de los significados de las acciones humanas y de la vida social. Se busca describir los fenómenos de la realidad sin la necesidad de buscar predecirlos. El alcance de la 
investigación es descriptivo exploratorio, dado que, si bien se busca describir un fenómeno, como es requisito para los estudios de tipo descriptivo (Hernández, Fernández y Baptista), no existen referencias contundentes sobre trabajos que utilicen los mismos conceptos teóricos que esta investigación, por tanto, tiene un carácter exploratorio.

\subsection{Corpus}

Las unidades de análisis consideradas en este estudio corresponden a comentarios posteados por lectores/as en páginas de Facebook de medios chilenos como son Chilevisión, CNN Chile y Cooperativa. Esta selección pertenece al corpus de una investigación más amplia, en el que se identificaron otras noticias acerca de situaciones relacionadas con personas LGBTIQ+, pero, para este caso específico, el análisis se centra únicamente en aquellas referidas al ingreso de estudiantes transgénero a establecimientos educacionales chilenos y el reconocimiento y la validación de su identidad de género.

Para identificar las noticias se utilizaron tanto el buscador general de Facebook como el propio de las diferentes páginas de prensa nacional, usando palabras clave "LGBTIQ+", “LGBT”, “trans”, “transgénero", “multigénero". Luego de una sistematización de estas noticias, el equipo definió los siguientes criterios: ser una noticia de los últimos dos años; que refiera a una persona o haya sido generada por una persona LGBTIQ+; que haga alusión al ingreso de estudiantes trans a establecimientos educacionales y/o al reconocimiento de su identidad de género; y que haya generado un número superior a 50 comentarios. El número de corte se definió luego de observar la frecuencia de interacciones en cada noticia y se consideró solo aquellas que fueran más productivas. Los comentarios de las noticias seleccionadas fueron capturados en una plantilla Excel que consideraba el contenido y sus réplicas -incluyendo enunciados, gifs, emoticones o emojis- y la fecha en que fueron emitidos, omitiendo los datos personales de quien realizaba la intervención. Finalmente, mediante la saturación teórica, el corpus quedó conformado por cuatro noticias pertenecientes a tres medios diferentes. 
Artículo. Paulina Maldonado Delgado, Paola Alarcón Hernández, Carolina Venegas Carrasco, Mabel Urrutia Martínez. "Ingreso de estudiantes trans a establecimientos educacionales chilenos: análisis de topoi en comentarios de Facebook"

Tabla 1. Corpus

\begin{tabular}{|l|l|l|l|}
\hline \multicolumn{1}{|c|}{$\begin{array}{c}\text { Medio y } \\
\text { código }\end{array}$} & \multicolumn{1}{|c|}{ Titular } & \multicolumn{1}{c|}{$\begin{array}{c}\mathrm{N}^{\circ} \text { de } \\
\text { comentarios }\end{array}$} \\
\hline $\begin{array}{l}\text { Chilevisión } \\
\text { CH-L1 }\end{array}$ & $\begin{array}{l}\text { Joven trangénero aceptada en Liceo 1 } \\
\text { llegó a su primer día de clases }\end{array}$ & $05 / 03 / 19$ & 1137 \\
\hline $\begin{array}{l}\text { CNN Chile } \\
\text { CNN-L1 }\end{array}$ & $\begin{array}{l}\text { Mamá de Arlén Aliaga: "Yo también viví } \\
\text { un proceso de negación, pero son } \\
\text { nuestros hijos y el amor es más fuerte" }\end{array}$ & $01 / 03 / 19$ & 312 \\
\hline $\begin{array}{l}\text { CNN Chile } \\
\text { CNN-LM }\end{array}$ & $\begin{array}{l}\text { Independencia contará con el primer } \\
\text { liceo multigénero del país }\end{array}$ & $04 / 10 / 19$ & 301 \\
\hline $\begin{array}{l}\text { Cooperativa } \\
\text { CPT-IN }\end{array}$ & $\begin{array}{l}\text { Instituto Nacional permitió usar jumper a } \\
\text { estudiantes transgénero }\end{array}$ & & \\
\hline
\end{tabular}

\subsection{Procedimiento de análisis}

El análisis siguió las siguientes etapas: A) Preanálisis, en la cual se organizó el material a analizar y se definió el plan para examinar los datos. B) Codificación, en esta etapa se realizó una codificación abierta del contenido, es decir, asignando códigos libres o in vivo, y se catalogaron los elementos. Al mismo tiempo, se codificaron elementos comunicativos propios del lenguaje multimodal como gifs y emojis o emoticones. C) Categorización, se organizaron y clasificaron las unidades obtenidas en base a criterios de diferenciación. El criterio fundamental de categorización es semántico, es decir, se agrupan las unidades por similitudes de significado. Específicamente, se realizó una exploración inicial del corpus más amplio. El establecimiento de temas de interés se fundamenta en que esa codificación arrojó como resultado que los comentarios manifestaban actitudes o bien a favor o bien en contra del ingreso de estudiantes trans a establecimientos educacionales, y/o el reconocimiento de su identidad de género, y de las personas LGBTIQ+ en general, mediante topoi. Los casos que no pudieron ser codificados fueron excluidos como se explica en la sección siguiente. 


\section{Resultados}

Los comentarios presentados a continuación se encuentran divididos en dos macrocategorías relacionadas con la postura de quien emite su opinión, implícita o explícita, con respecto a las noticias. En primer lugar, se considerarán los comentarios con una actitud negativa frente al ingreso de estudiantes trans a los establecimientos educacionales y el reconocimiento de su identidad de género en los mismos (en contra) y luego aquellos que presenten una actitud positiva al respecto (a favor).

De un total de 2182 comentarios, solo el $28,1 \%$ manifiestan una postura favorable con respecto a las noticias, mientras que el $67,5 \%$ una postura en contra y el 4,3\% una indeterminada. Se clasificaron como indeterminados aquellos comentarios que no pudieron ser codificados con certeza o que se dirigían a personas cuyos comentarios ya no estaban disponibles al momento de seleccionar las noticias.

\subsection{Topos como recurso argumentativo en comentarios en contra}

En los comentarios que manifestaban una postura en contra de las noticias acerca del ingreso de estudiantes transgénero o transexuales a establecimientos educacionales, se lograron identificar seis topoi, de los cuales tres fueron adaptados de los mencionados por Wodak $(116,117)$, en su análisis de discursos políticos y discriminatorios, y tres restantes identificados particularmente en los comentarios de las cuatro noticias en torno a las que se desarrolla este análisis.

El topos de definición o interpretación -Locus a nominis interpretatione- es descrito por Wodak mediante la regla conclusiva de que si una acción, cosa, persona o grupo de personas es designado o nombrado de una determinada manera debería poseer las cualidades, rasgos y/o atributos propios de este nombre o que son parte del significado literal de este. En el caso de las noticias que aluden al reconocimiento de estudiantes transgénero en establecimientos educativos, la argumentación está basada en una declaración condicional: "si quiere ser mujer debe verse y actuar como una". Considerando los comentarios, esta condición está compuesta por dos dimensiones que socialmente se 
Artículo. Paulina Maldonado Delgado, Paola Alarcón Hernández, Carolina Venegas Carrasco, Mabel Urrutia Martínez. "Ingreso de estudiantes trans a establecimientos educacionales chilenos: análisis de topoi en comentarios de Facebook"

encuentran relacionadas pero que son diferentes: la genitalidad y los roles y estereotipos de género.

En el primer caso, los comentarios apuntan a que una mujer para ser considerada como tal debe poseer características físicas relacionadas con su biología que evidencien su sexo. Es por esto que se estima correcto y necesario realizar intervenciones quirúrgicas para corregir esta situación. Sería solo a partir de entonces que el cambio de género obtendría validez, como se evidencia en la siguiente muestra de comentarios ${ }^{2}$ :

- Chicos no basta con creerse debes también ser... En otros países se operan... Así no encontraremos "'hombres"" embarazados.... Cambió de nombre y operación obligatoria.... (CHV-L1)

- Este idiota cree que con vestido es mujer, si quiere serlo que se opere (CHVL1)

- Me pregunto y sus papas. El joven tiene pene. Y se cree mujer, lo mejor es operarse. Y cortarles lo masculino y ser mujer. (CHV-L1)

En el segundo caso, las exigencias propuestas para reconocer el género de una persona apuntarán hacia los roles del género, es decir, cómo socialmente se espera que actúe una persona de un género determinado. Esta visión se enmarca en conductas y apariencias estereotipadas con las que se relaciona al género femenino en la cultura occidental.

- Pero si quiere ser mujer que se comporte como tal... porque con la facha que tiene no calienta a nadie. (CHV-L1)

- No tiene ni un rasgo de transgenero. El es un hombre vestido de mujer y cochino mas encima. Las uñas asquerosas puag (CHV-L1)

- Que pendejadas x la xuxa, mínimo queris ser mujer, creete el cuento arreglate peinate y maquillate $\mathrm{x}$ lo menos $\mathrm{q}$ atroz $\square q(\mathrm{CHV}-\mathrm{L} 1)$

\footnotetext{
${ }^{2}$ Se procuró mantener la forma original de cada comentario, haciendo correcciones exclusivamente cuando la redacción dificultaba la comprensión y eliminando los nombres de las personas a las que algunos estaban dirigidos.
} 
- Mínimo una encrespadita de pestañas, una pintadita poca para no parecer hombre, xq su cara es muy de hombre (CHV-L1)

El topos de ley natural o naturalidad se encuentra en comentarios que manifiestan una postura negativa frente a las noticias sobre el ingreso de estudiantes transexuales y/o transgénero a establecimientos educacionales. Este topos basa su argumentación en la biología y se rige por la condicional de que si los seres humanos nacen con determinados rasgos que definen su genitalidad, esto no puede ni debe ser modificado.

Se podría considerar que este topos es incongruente con el de definición, en el que el cambio de sexo es una exigencia por parte de las personas en contra. La diferencia se encuentra en que, en ambos, las opiniones apuntan hacia aspectos diferentes. Si bien en el de naturalidad se hace referencia a la genitalidad, es decir, a un aspecto físico, lo realmente fundamental es la exigencia de que la identidad de género y el sexo biológico deben coincidir y que cualquier situación en la que esto no se mantenga es errónea y debe ser corregida, pero no a un nivel físico sino que psicológico. En este caso, no se apunta a los roles y estereotipos de género explícitamente sino que, por el contrario, el factor biológico o físico es el que naturalmente determinaría las demás dimensiones. En el caso del topos de definición, el físico debe adecuarse al género, por lo que los cambios corporales son permitidos, mientras que en el de naturalidad es al revés, lo admitido es una adecuación psicológica, no física.

- enferma es esa gente q cree q un hombre se puede convertir en mujer de la nada! Y q todos ignoren q su aparato reproductor puede ser remplazado o cambiado!! (Cosa q nunca pasara) esa gente para mi! Esta loco y enferma!!! (CHV-L1 n ${ }^{\circ} 4$ 19)

- evolucionar a que a que los pervertidos sean moda, estas muy mal xx mujer xy hombre el cromosoma del maricon no existe (CHV-L1 n 28-13)

- WTF ahora van los travestis al colegio que chuchaaaaaa... Si tiene pene es hombre y punto, en mi opinión es tolerable que sean gay ya eso pasable, pero que mierda está moda qla enferma, no se dan cuenta que son repulsiv@s????? (CHV-L1 n ${ }^{\circ}$ 95) 
Artículo. Paulina Maldonado Delgado, Paola Alarcón Hernández, Carolina Venegas Carrasco, Mabel Urrutia Martínez. "Ingreso de estudiantes trans a establecimientos educacionales chilenos: análisis de topoi en comentarios de Facebook"

- Debe tener las bolas hinchadas tanto comentario a favor y en contra el tema es sencillo en su cédula nacional aparece como masculino. Fin de la historia $\left(\mathrm{CNN}-\mathrm{L} 1 \mathrm{n}^{\circ} 84\right)$

El topos de peligro se basa en que si determinadas acciones implican consecuencias negativas o que pueden significar una amenaza, estas no deberían ser realizadas. Wodak considera que mediante este esquema argumentativo se pueden invertir las posiciones entre victimario y víctima, debido a que de esta manera la responsabilidad de los prejuicios recaería sobre las últimas. El foco principal del temor expresado ante el ingreso de una estudiante transgénero se encuentra en la actitud que ella podría adoptar frente a sus compañeras de establecimiento. En este caso, a las personas trans se les asigna un perfil peligroso, percibido por parte de los/as comentaristas en contra.

- Después se acordará a es hombre y Capaz q se viole a sus compañeras Porque la mayoría de la gente homosexual tienen una mente depravada (CHV-L1)

- Y cómo se q se está aciendo el listo para espiar a las niñas (CHV-L1)

- Cuando las tenga a todas atravesa los quiero ver (CHV-L1)

- Seria raro que ninguna terminara embarazada del trans (CHV-L1)

- Fin de año, tres compañeras embarazadas (CHV- L1)

El topos de la justicia está basado en la exigencia de derechos iguales para todas las personas, lo que se resume en la condicional de que "si las personas, las acciones o las situaciones son iguales en determinados aspectos concretos, deben recibir el mismo trato o ser objeto de la misma consideración" (Wodak 117). En este sentido, la argumentación se sostiene en que si las solicitudes de las personas trans se relacionan con el reconocimiento de los mismos derechos, entonces no deben gozar de ningún tipo de ventaja con respecto a los/as demás. En lo que a ventajas refiere, en este caso, es considerada como tal haber ingresado a un establecimiento mediante un proceso de admisión especial, motivado por las denuncias de bullying en centros anteriores. 
- Se supone que la ley dice que todos los seres humanos nacen libres en igualdad y derechos. Cosa que aqui no se cumple, pasa a llevar a las niñas que estan en el liceo y aquellas que estan en lista de espera... solo por su "condicion sexual" que injusto no lo creen. (CNN- L1)

- Ya me tienen bien aburrida estos trans .. se pasaron por la raja .. el protocolo de matrícula. Pasan a llevar al resto .. si quieren respeto empiezen de respeto .. pero estos homo están muy sublevados $(\mathrm{CNN}-\mathrm{L} 1)$

- Ninguna niña ya casi en marzo podría haber entrado al liceo 1 aunque fuera su sueño ! Pero este joven si lo logro será porque hoy en día las minorías tienen un mayor apoyo me parece que es muy injusto para las niñas que dieron el examen y quedaron en la lista de espera (CNN- L1)

- Ahora en chile los trans, gay y lesbianas mandan que paso con las otras chicas que quedaron sin cupo se las metieron en la raja (CNN- L1)

- Muy persona sera como dijeron por ahi hay niñas que no pueden hacer eso y tienen mas meritos academicos que el para quedar en ese liceo, pero parece que eso no importa si no pertenecen a una minoria escandalosa (CNN- L1)

En el topos de advertencia se consideran aquellos comentarios que buscan alertar a las/os lectores/as acerca de lo que implican estos hechos, mencionados en las noticias, para el futuro de la sociedad chilena. A diferencia del topos de peligro, en el de advertencia no se hacen proyecciones en términos particulares de la amenaza que podría representar la presencia de una estudiante transgénero. Este topos apunta, de una manera un tanto profética, hacia las repercusiones deberá enfrentar el país, en términos sociales y morales, si se permiten estos actos.

- Q obscenidades permiten las autoridades q se espera a futuro quizas. Q. Perversidades bs a autorizar q terrible (CHV-L1 $\left.\mathrm{n}^{\circ} 137\right)$

- El mundo al revés, niños con faldas, niñas con el pelo al rape, y se está cumpliendo la profecía de la biblia " vendrán tiempos peores". (CNN-LM $\left.n^{\circ} 113\right)$ 
Artículo. Paulina Maldonado Delgado, Paola Alarcón Hernández, Carolina Venegas Carrasco, Mabel Urrutia Martínez. "Ingreso de estudiantes trans a establecimientos educacionales chilenos: análisis de topoi en comentarios de Facebook"

- Pobre humanidad que lastima que acepta tanta aberración como normal (CNNL1 $n^{\circ} 9$ )

- Estamos en una profunda crisis social y moral aca en Chile....el concepto de familia se esta yendo al carajo.somos subdesarrollado y nos creemos con una mente amplia no hay que permitir estas aberraciones el hombre es hombre y la mujer es mujer...no ecisten los híbridos. (CHV-L1 n¹02)

El topos de irrelevancia se distingue en aquellos comentarios que desarrollan dos posturas en torno a las noticias sobre el ingreso de estudiantes transgénero a determinados establecimientos educativos o el reconocimiento de la identidad de género de los/as mismos/as en estos centros. En primer lugar, se encuentran aquellas personas que consideran sin importancia los hechos presentados en las noticias, actitud que es demostrada implícitamente mediante enunciados con fines humorísticos (CNN- L1) o de manera explícita. En segundo lugar, se presentan comentarios que apuntan hacia que los recursos e innovaciones de los colegios deberían estar destinados a otros fines, debido a que existen falencias mucho más relevantes que deberían ser prioritarias.

- Por qué marcan su condición sexual ?Que les vaya bien a todos en su año escolar ... ni ahí que sea transexual . (CHV-L1)

- Bueno mi gente quiero aprovechar este $1 \%$ de bateria para dar mi opinión yo pienso que cuan (CNN-LM)

- En vez de hacerlo inclusivo en general, lo hacen "multigenero" que es esa wea, hay niños con Sindromes, movilidad reducida u otros tipos de condiciones o enfermedades que no pueden ingresar a colegios cercanos porque aún no tienen los profesionales o adaptaciones necesarias para ellos. Pero bueno, qué más vamos a pedir, si en este país legislan pa la galucha ideológica y no por las necesidades reales. (CNN-LM)

- En vez de concentrarse en mejorar la calidad de la educación en estos liceuchos se enfocan en aceptar a cualquier desviados, así de cagados estamos (CNN-LM) 


\subsection{Topoi como recursos argumentativos en comentarios a favor}

En los comentarios que manifestaron una postura a favor o positiva con respecto a las noticias acerca del ingreso de estudiantes trans a establecimientos educacionales y el respectivo reconocimiento de su identidad de género, se distinguieron dos tipos de topoi.

En el primero, que corresponde al topos de Evolución y progreso, se considera que los hechos expuestos en las noticias son positivos ya que significan un avance necesario para la sociedad chilena. Desde esta perspectiva, se mantiene la idea de que quienes se manifiesten en contra son personas que necesitan actualizar sus conocimientos, ya que sus concepciones, en cuanto a lo que el sexo y el género respectan, no se encuentran en concordancia con la realidad.

- Claramente ya es mixto si las chicas están allí estudiando. Muy bien!! A

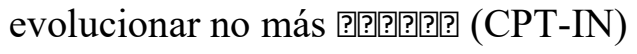

- Los únicos que podrían opinar sobre esto son los propios alumnos, y está claro que las nuevas generaciones son más evolucionadas que todos los que se hacen los chistosos desde el celular. (CPT-IN)

- si sabía....es una buena noticia....ojalas todos los colegios avanzaran asi!!!! (CNN-LM)

- Esto demuestra evolución social, evidentemente los simios no lo entienden! (CPT-IN)

El segundo topos identificado es el de Respeto por la libertad y la diversidad. Este apela a que toda persona, sin importar su orientación sexual o identidad de género, tiene el derecho de desenvolverse libremente en sociedad y merece tanto respeto como cualquier otra. Estos comentarios se caracterizan también por interpelar a quienes manifiestan una postura en contra, señalándoles que el tema en discusión no es algo que les pueda afectar negativamente, por lo que su opinión no sería pertinente.

- En que les afecta tropa de idiotas? Lo usarán uds.? No! Vivan y dejen vivir que ningún daño les hacen (CPT-IN) 
Artículo. Paulina Maldonado Delgado, Paola Alarcón Hernández, Carolina Venegas Carrasco, Mabel Urrutia Martínez. "Ingreso de estudiantes trans a establecimientos educacionales chilenos: análisis de topoi en comentarios de Facebook"

- Wea de cada uno lo que haga, por que chucha se sienten con el derecho de opinar sobre lo que haga otra persona, esto ni noticia debiese de ser, vivan su vida! (CPT-IN)

- para mi todas las personas son iguales cada uno sabe como vive su vida, lo importante es ser feliz ...... (CHV-L1)

- Es efectivo, yo ayer vi a una chica con jumper del instituto, debo admitir que fue raro, no obstante todo mi respeto...eso es lo que debe prevalecer el respeto por la.diversidad... (CPT-IN)

Además, en este topos se presentaron de manera reiterada enunciados que apuntan directamente hacia quienes manifiestan una postura en contra, con el objetivo de hacerles reflexionar acerca de lo que ocurriría si alguien cercano/a a ellos/as tuviera que enfrentarse a ese tipo de comentarios. Estos enunciados buscan advertir y hacer un llamado a la empatía y el respeto desde la premisa principal de que cualquiera podría atravesar esta situación.

- Irrespetuoso indolente, quizás el día de mañana, tus hijos o nietos también pasen por la crueldad que tu provocas a los demás. Piensa un poco, antes de hablar estupideces por hacerte el payaso. (CHV-L1)

- Que triste que te burles de una niña en cuerpo equivocado, cuando tienes una HIJA tan pequeña y después pedirás respeto para ella, ojala nadie se burle de ella como lo haces (CHV-L1)

- Pensar que despues se conmueven por las historias y suicidios por el bullying ojala a sus hijos nunca le pase que una manga de estúpidos se burle por su condicion sexual o por su forma de ser gente estupida como se sentira ella al ver como todos se burlan por su condicion weon a nadie le gustaria que le hicieran esa wea (CHV-L1) 


\subsection{Discusión}

Como quedó en evidencia, los comentarios que manifiestan una actitud positiva con respecto al ingreso de estudiantes transgénero a establecimientos educacionales chilenos son escasos en comparación con los que sostienen una actitud negativa. El argumento predominante que justifica una postura a favor es el de respeto por la libertad y la diversidad, debido a que estos enunciados en su mayoría apuntan a que toda persona merece gozar de los mismos derechos que las demás y desenvolverse libremente en sociedad. En este caso específico, se traduce en el reconocimiento y aceptación de su identidad de género. Por este motivo, las noticias son concebidas como hechos positivos que representan avances para el país en materias de inclusión y derechos humanos.

Si bien en la categoría de comentarios en contra se identificaron seis topoi, existen enunciados que describen globalmente los argumentos fundamentales bajo los que se justifica una postura negativa con respecto al ingreso de estudiantes trans a los establecimientos. En este contexto, se encuentra la afirmación de que el género y el sexo biológico deben coincidir, es decir, una persona con características biológicas femeninas debe en consecuencia presentar rasgos y actitudes asociadas a la feminidad.

En este sentido, la aceptación y el respeto de la identidad de género de personas trans es considerada, por quienes comparten la postura en contra, una condición antinatural. A partir de esto, surge la idea de que el ingreso de estudiantes trans a establecimientos educacionales representa un peligro para los demás miembros de la comunidad escolar, ya que su antinaturalidad significa una amenaza para el bienestar y la integridad física y psicológica del resto de niños, niñas y adolescentes con quienes se relacionen. Utilizando estos argumentos como justificación, comentaristas que manifestaban una postura en contra consideraban que cualquier reacción violenta por parte de otros/as estudiantes era válida y necesaria.

Como ya fue mencionado, en los comentarios se pueden identificar claramente dos posturas ideológicas opuestas, en relación con las noticias que forman parte del corpus. Sin embargo, existe una coincidencia en ambas posturas, que para este estudio cobra especial relevancia, relacionada con la falta de información y las confusiones conceptuales en temas referidos al sexo y el género. Las afirmaciones más frecuentes son aquellas en que se 
Artículo. Paulina Maldonado Delgado, Paola Alarcón Hernández, Carolina Venegas Carrasco, Mabel Urrutia Martínez. "Ingreso de estudiantes trans a establecimientos educacionales chilenos: análisis de topoi en comentarios de Facebook"

establece una equivalencia por parte de los/as comentaristas entre la identidad de género, la expresión de este y la orientación sexual de una persona. Por ejemplo, en comentarios con una postura positiva frente a las personas trans, este aspecto se puede identificar en expresiones como "ojala No Tengas Un Hijo Gay!" (CHV-L1) o "que feo como se refiere a las personas homosexuales. Porque ellos son personas común y corriente" (CHV-L1). Mientras que en comentarios con una actitud negativa se presentan enunciados como "solo existen 2 géneros Lo demás son preferencias sexuales" (CNN-LM) o "nadie nace maricon ni lesbiana" (CHV-L1).

Estos y otros resultados obtenidos, sumados a los casos de homofobia y transfobia que han debido enfrentar personas LGBTIQ+ en el contexto escolar (Movilh, XVIII; XI), nos parecen relevantes al momento de abordar las discusiones en torno al impacto de estas situaciones en materia educativa. Teniendo esto en cuenta, el objetivo de esta investigación, analizar los topoi en los que se basan las posiciones a favor y en contra del ingreso de estudiantes trans a establecimientos educacionales chilenos, surge a partir del interés por comprender las situaciones que podrían enfrentar personas LGBTIQ+ dentro del contexto educativo.

El Ministerio de Educación, en su documento Oportunidades curriculares para la educación en sexualidad, afectividad y género (MINEDUC 7), señala que es responsabilidad del estado apoyar y orientar a los establecimientos educacionales "para que puedan abordar la formación en sexualidad, afectividad y género con sus estudiantes" (7) a través de un proceso que asegure el acceso a educación oportuna, sin sesgo y con bases científicas. Sin embargo, en el mismo documento se declara que este apoyo debe garantizar la autonomía de los establecimientos con un plan acorde a su propio Proyecto Educativo.

Esta última afirmación nos resulta ambigua ya que, si bien respetar la diversidad de cada colegio, escuela o liceo es necesario, al mismo tiempo es innegable que existen establecimientos con miradas ideológicas y valóricas muy marcadas que a veces pueden interferir en el abordaje libre y objetivo de estas temáticas. Complementando esta idea, la investigación "Vivencias de les jóvenes transgénero respecto a su inclusión social en Chile" revela que la generalidad de las personas entrevistadas no percibía la escuela como un espacio seguro para expresar su identidad de género con libertad, además de señalar que 
han "sufrido discriminación y algún tipo de violencia, asociando estos sucesos a la escasa tolerancia, educación y políticas públicas de resguardo de derechos" (Fischer et. al. 25).

Todos los datos presentados evidencian la necesidad de adoptar políticas más estrictas y actualizadas en relación con la educación en género y sexualidad, para garantizar el bienestar no solo de los/as estudiantes, sino de toda la comunidad escolar y, en consecuencia, de la sociedad en general. Esto debido a que una conducta violenta o discriminatoria en el contexto escolar puede afectar a niños, niñas, adolescentes, pero también a apoderados/as, docentes o cualquier miembro del personal del establecimiento. Tal como señalan Mardones et. al., la prevalencia del discurso binario y heteronormativo en las políticas de educación chilena debe ser reflexionada y deconstruida a fin de permitir un acceso a espacios educativos abiertos y heterogéneos que acojan la pluralidad de manifestaciones de la sexualidad.

\section{Conclusiones}

Tras el análisis del corpus se identificaron dos posturas ideológicas opuestas evidentes por parte de los/as comentaristas con respecto al ingreso de estudiantes transgénero a establecimientos educacionales chilenos, siendo cada una respaldada por diferentes topoi. Desde el marco de la Lingüística Cognitiva, los resultados muestran que el análisis discursivo de los medios de comunicación permite evidenciar las formas en que las personas construyen y negocian representaciones socio-cognitivas, las que comprenden un conglomerado de conceptualizaciones, actitudes, valoraciones y emociones que pueden tener un impacto en conductas que pueden ser reivindicativas de las libertades individuales o, lamentablemente, estar en la base de conductas de discriminación y menoscabo hacia las personas LGBTIQ+.

En concordancia con lo que señala Sal Paz (Comentario), en los comentarios analizados, las secuencias textuales son predominantemente argumentativas, a pesar de presentarse modos de organización textual narrativos, descriptivos y expositivos. Por este motivo, distinguir las estrategias argumentativas o topoi (Wodak) resultó relevante para comprender el razonamiento principal en torno al que se desarrolla cada postura. Además, en la medida en que los topoi corresponden a lugares comunes en la argumentación 
Artículo. Paulina Maldonado Delgado, Paola Alarcón Hernández, Carolina Venegas Carrasco, Mabel Urrutia Martínez. "Ingreso de estudiantes trans a establecimientos educacionales chilenos: análisis de topoi en comentarios de Facebook"

(Ibarra), ya que suponen una creencia compartida por una colectividad (Ascombre y Ducrot), su estudio contribuye a revelar la forma en que un hecho o grupo de personas es percibido socialmente.

Considerando la dinámica bajo la que funcionan los comentarios en Facebook, muchos de estos comenzaban abordando el tema específico de la noticia, pero, mediante las interacciones, la discusión era dirigida a tópicos diferentes. Por este motivo, una de las proyecciones de este estudio es profundizar en las representaciones discursivas del ser hombre o ser mujer y su relación con el discurso transfóbico en Chile, teniendo en consideración que los estereotipos de género podrían visualizarse de manera distinta según el tipo de red social o plataforma de la que se extraiga el corpus.

\section{REFERENCIAS}

Ascombre, Jean-Claude y Oswald Ducrot. La argumentación en la lengua. Editorial Gredos, 1994.

Ascombre, Jean-Claude. "Semántica y léxico. Topoi, estereotipos y frases genéricas". Revista Española de Lingüística, vol. 25, no. 2, 1995, pp. 297-310.

Augoustinos, Martha, Iain Walker, Ngaire Donaghue. Social Cognition: An Integrated Introduction, 2da ed. SAGE, 2006.

Catamutto, Lucía y Cristina Vela Delfa. "El discurso digital como objeto de estudio: de la descripción de interfaces a la definición de propiedades". Aposta. Revista de Ciencias Sociales, vol. 69, 2016, pp. 296-323.

Croft, William. "Toward a social cognitive linguistics". New Directions in Cognitive Linguistics, edición de Vyvyan Evans y Stéphanie Pourcel. Ámsterdam, John Benjamins, pp. 395-420.

Dąbrowska, Ewa y Dagma Divjak, eds. Handbook of Cognitive Linguistics. De Gruyter Mouton, 2015.

De Piero, José Luis y María Evangelina Narvaja. "Representaciones de género en comentarios digitales en dos Fan Page de Facebook". Revista Latinoamericana de Ciencias Sociales, Niñez y Juventud, vol. 16, núm.2, 2018, pp. 759-775.

Fischer, Fernanda et. al. "Vivencias de les jóvenes transgénero respecto a su inclusión social en Chile". Revista Nomadías, núm. 27, 2019, pp. 9-31.

García, Víctor y Liliana Gutiérrez, editores. Manual de géneros periodísticos. Ecoe Ediciones, 2011.

Hernández-Sampieri, Roberto, Carlos Fernández y Pilar Baptista. Metodología de la investigación. McGraw Hill, 2010.

Ibarra, Daniela. "Topoi como recurso argumentativo en comentarios de blogs de tema político en ciberperiódicos chilenos". Revista Latinoamericana de Estudios del Discurso, núm. 18, núm. 2, 2018, pp. 59-76. 
Koller, Veronika. "How to Analyse Collective Identity in Discourse-Textual and Contextual Parameters". Critical Approaches to Discourse Analysis across Disciplines, vol. 5, núm. 2, 2012, pp. 19-38.

Landau, Mark, Michael Robinson y Brian Meier, eds. The power of metaphor: Examining its influence on social life. American Psychological Association, 2014.

Mardones, Karen, Marcela Apablaza y Pamela Vaccari. "Binary discursivities in educational policies on gender and sexuality in Chile". Estudios pedagógicos, vol. 46, núm. 1, 2020, pp. 399-411.

Meneses, Alejandra. "Leer y escribir en una escuela chilena: Representaciones discursivas de los diferentes agentes educativos en las áreas prioritarias del currículo escolar en NB3”. Revista Signos, vol. 41, núm. 67, 2008, pp. 257-278

Ministerio de Educación (MINEDUC). Oportunidades curriculares para la educación en sexualidad, afectividad y género. MINEDUC, 2018.

Montecino, Lésmer. "Cortesía, ideología y representaciones discursivas en la gestión conversacional de jóvenes chilenos." Onomázein, vol. 12, 2005, pp. 9-22.

---. "Personas en situación de calle en Santiago de Chile: identidad y futuro". Discurso \& Sociedad, vol. 2, núm. 2, 2008, 330-356.

Moscovici, Serge. El psicoánalisis, su imagen y su público. Huemel, 1979 (Edición en francés, 1961).

Movimiento de Integración y Liberación Homosexual (Movilh). XVIII Informe Anual de Derechos Humanos de la Diversidad Sexual y de Género en Chile. Movilh, 2020.

---. XIX Informe Anual de Derechos Humanos de la Diversidad Sexual y de Género en Chile. Movilh, 2021.

Ramírez, Óscar y Laura Valencia. Discurso y prensa: nominación de los actores sociales y los hechos en las noticias sobre violencia contra la mujer. 2017. Universidad Tecnológica de Pereira, trabajo de grado realizado para obtener el título de Magíster en Lingüística.

Ramírez-Lasso, Lilia. "Representaciones discursivas de las migrantes venezolanas en medios digitales". Revista Latinoamericana de Estudios del Discurso, vol. 18, núm. 2, 2018, pp. 42-58.

Romano, Manuela y María Dolores Porto. "Discourse, cognition and society". Manuela Romano, María Dolores Porto, eds. Exploring discourse strategies in social and cognitive interaction.John Benjamins Publishing Company, 2016, pp. 1-17.

Sal Paz, Julio. "Comentario Digital: género medular de las prácticas discursivas de la cibercultura". Caracteres. Estudios culturales y críticos de la esfera digital, vol. 2, núm. 2, 2013, pp. 152-171.

Sal Paz, Julio. El comentario digital como género discursivo periodístico. Análisis de la Gaceta de Tucumán. Aposta: Revista de ciencias sociales, vol. 69, 2016, pp. 158216.

Van Dijk, Teun. Society and discourse. How social contexts influence text and talk. Cambridge University Press, 2009.

Wodak, Ruth. "El enfoque histórico del discurso". Métodos de análisis crítico del discurso, compilación de Ruth Wodak y Michael Meyer. Gedisa, 2003, pp. 101-142 